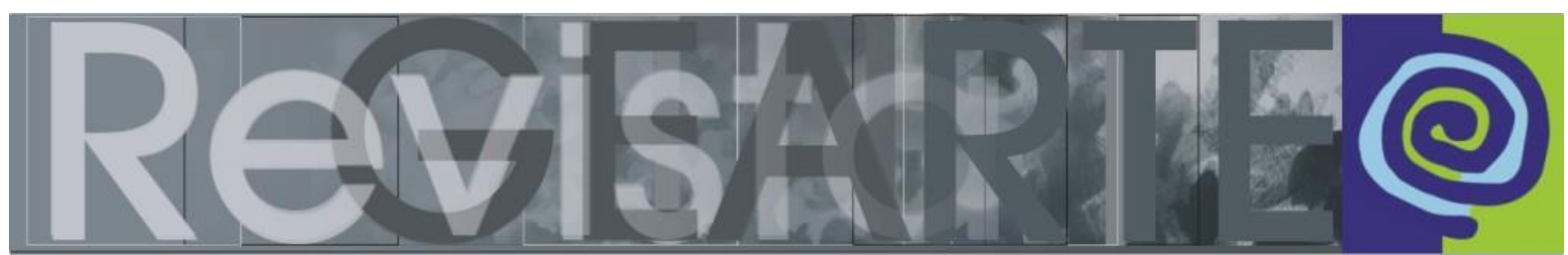

e-ISSN 2357-9854

\title{
Aprendizagem Colaborativa Online e Educação Artística
}

\author{
Vitor Silva (Escola Básica Integrada de Lagoa - EBI LAGOA, Lagoa, Portugal) \\ Clara Coutinho (Universidade do Minho - UMINHO, Braga, Portugal)
}

\begin{abstract}
RESUMO - Aprendizagem Colaborativa Online e Educação Artística - Este artigo apresenta um estudo de caso efetuado com alunos do ensino fundamental analisando as atividades colaborativas realizadas numa comunidade criada na plataforma digital Edmodo. A técnica de desenvolvimento da investigação posicionou-se no paradigma interpretativo e qualitativo recorrendo à análise de conteúdo das discussões e dos artefactos criados. Os resultados mostram o nível de colaboração do grupo e a influência de algumas ferramentas e métodos de trabalho, tornando evidente a necessidade de realização de outros estudos sobre comunidades análogas dedicadas à educação artística em faixas etárias precoces.
\end{abstract}

PALAVRAS-CHAVE

Do-It-Yourself. Edmodo. Aprendizagem Colaborativa Online. Metodologia Projetual.

ABSTRACT - Collaborative Online Learning and Art Education - This paper presents a case study carried out with elementary school students analyzing the collaborative activities developed in a community created in the Edmodo digital platform. The research development technique was positioned in the interpretative and qualitative paradigm using the content analysis of the discussions and artifacts created. The results show the level of collaboration of the group and the influence of some tools and methods, making evident the need to do other studies on analogous communities dedicated to artistic education in the early age.

KEYWORDS

Do-It-Yourself. Edmodo. Online Collaborative Learning. Project Methodology.

\section{Introdução}

Os vídeos e as apresentações tutoriais que na Internet nos ensinam a realizar tarefas várias, desde as mais triviais às mais complexas, são hoje um hábito e os jovens não estando alheios a estas transformações envolvem-se de forma contínua e mais ou menos consenciente em contextos de aprendizagem colaborativa.

Partindo desta constatação empírica e tendo em conta a escassez de estudos e investigações realizados sobre estas matérias, particularmente em idades precoces, empreendemos uma investigação com o intuito de aferir de que forma a criação de uma comunidade de aprendizagem que incidisse neste tipo de conteúdos poderia estimular a aprendizagem colaborativa, mais especificamente com alunos do $5 .^{\circ}$ ano de escolaridade. 


\section{O fenómeno DIY}

O acrónimo DIY (Do-It-Youself), ou faça-você-mesmo, surge em meados do século XX, referindo-se ao ato de criar, produzir, modificar ou reparar algo sem o apoio de profissionais, já a sua génese ideológica é normalmente associada ao movimento punk, às suas fanzines e música de produção própria, feita com meios parcos e com uma mensagem subversiva. Hoje tem significado amplo em carácter e alcance, descrevendo uma vasta gama de atividades e comportamentos, desde as fablabs em que se criam e projetam novos produtos, aos artesãos urbanos que ganham a vida através do Etsy ou ainda a práticas de ensino autodirigido (KAMENETZ, 2010).

Apesar de se apresentar como uma reação à normalização, à estandardização e ao capitalismo, e de encontrar forte adesão em movimentos contracultura, esta prática tem vindo a generalizar-se, e a sair fora dos nichos a que estava tradicionalmente associada na mesma medida que se expande em comunidades no mundo virtual. Os conteúdos DIY na Internet construídos por crianças e jovens, nomeadamente em canais do Youtube, são hoje uma realidade bem presente, que advém da possibilidade de através deles aprenderem, exprimirem e mostrarem à escala global aquilo que são capazes de fazer. A proliferação de conteúdos multimédia deste tipo não é alheia à facilidade de acesso à internet, à portabilidade dos meios de produção/edição e ao uso fácil e intuitivo destas ferramentas. Com efeito, Kafai \& Peppler (2011) referem que os educadores devem estar especialmente interessados nas comunidades DIY, dada a quantidade de tempo que os jovens aí gastam voluntariamente em intensa aprendizagem. Tradicionalmente, os investigadores e profissionais da educação têm-se centrado no desenvolvimento da compreensão crítica dos média como um aspeto fundamental da literacia digital, no entanto as pesquisas têm evoluído notando-se que os jovens de hoje tanto consomem conteúdos ao navegar na Internet, como também partilham essas informações em sítios e redes sociais contribuindo em blogues, realizando animações ou criando artefactos gráficos e produções de vídeo (ITO et al., 2009).

Esta perspetiva de que a produção de conteúdos multimédia online é um sobrepor de camadas e influências que se mobilizam através do trabalho colaborativo, também encontra eco na mutação que alguns autores fazem do termo Do-It-Yourself. 
Jenkins (2009) fala em Do-It-ourselves ou Do-It-Togheter, já Ackerman (2013) propõe que estamos em trânsito do DIY para o BIIT (Be In It Togheter).

No estudo "Rise of the expert amateur, DIY projects, communities and cultures" Stacey Kuznetsov e Eric Paulos, analisam seis destas comunidades - Etsy, Instructables, Crafster, Raverly, Dorkbot e Adafruit - concluindo que nelas se manifesta um "conjunto único de valores, enfatizando a partilha aberta, a aprendizagem e a criatividade sobre o lucro e o capital social" (KUZNETSOV \& PAULOS, 2010, p. 1).

Um bom exemplo do fenómeno em idades precoces é o sítio DIY.org, onde as crianças e jovens na menoridade são convidados a partilhar saberes, participando em projetos, respondendo a desafios e demonstrando as suas habilidades e perícias através de vídeos tutoriais.

\section{A aprendizagem colaborativa online}

A aprendizagem colaborativa tem-se estruturado e desenvolvido a partir de diferentes modelos e abordagens cujo traço comum é a pré-existência de mecanismos de interação que possam promover a colaboração. Um ambiente de aprendizagem com instrumentos de interação poderosos não garante no entanto que colaboração venha a ocorrer, para tal devem ser tomadas ativa e conscientemente um conjunto de medidas específicas, que partem da compreensão do próprio conceito, seguido do entendimento e do reconhecimento de como a colaboração se pode manifestar em contexto online (LISBÔA \& COUTINHO, 2013).

O conjunto de atividades coordenadas que os processos colaborativos implicam resulta da tentativa de construir e manter de forma sustentada uma conceção partilhada de um problema, manifestando-se numa teia de relações intencionais que resulta na urgência de produzir algo em conjunto, seja resolver um problema, seja criar ou descobrir alguma coisa, envolvendo um trabalho combinado para atingir objetivos comuns (DILLENBOURG, 1999).

Roschelle e Teasley (1995) definem o espaço do problema partilhado como uma estrutura de conhecimento comum que suporta a resolução de problemas através 
da integração de objetivos, das descrições do estado atual do problema, e da consciência de estratégias potenciais para o resolver, bem como as ligações entre esses fatores.

Como explicam Van Boxtel, et al. (2000), as atividades de aprendizagem colaborativa em que os alunos fornecem explicações aos seus pares sobre as suas ideias, podem ajudá-los a elaborar e reorganizar os seus conhecimentos, sendo muitas vezes através do intercâmbio verbal que o entendimento dos conceitos é visível. Os alunos entram em processos de negociação o que significa chegar à convergência, ou à compreensão e à criação partilhada de artefactos.

\section{O Edmodo}

O Edmodo é uma plataforma de ensino a distância que é uma espécie de híbrido de sistema de gestão de aprendizagens e rede social desenhado para a Educação. Tem como vantagens ser seguro, livre e estar traduzido em Português, a interface é simples, intuitiva e semelhante a do Facebook, o que facilita a ambientação e a interação entre os usuários. Não existe uma idade mínima de acesso estipulada, já que o cadastro é mediado por pais e professores.

A operacionalização das atividades baseia-se na criação de grupos aos quais os alunos acedem através de um código de registro. As atividades desenrolam-se integralmente através do sítio na Internet sendo possível partilhar conteúdos de sala de aula e convidar os alunos a realizar tarefas. Todas as interações ficam registradas num painel central e cada grupo tem uma biblioteca própria, onde podem ser armazenados recursos, imagens e vídeos. A biblioteca também faz a coleta automática dos recursos que toda a turma vai criando e publicando. Os alunos podem ser distinguidos com emblemas que reforçam positivamente uma ampla gama de comportamentos.

A plataforma abrange os principais agentes educativos, os encarregados de educação podem acompanhar as atividades a partir de um código de acesso específico fornecido pela conta do educando, para além de salas de aula virtuais, é possível criar páginas de escola e até de distrito, com as necessárias autorizações. 
Para garantir a facilidade e portabilidade no seu uso, a plataforma é compatível com telemóveis e outros dispositivos móveis.

\section{Metodologia}

A comunidade de aprendizagem online que criamos desenvolveu-se no Edmodo ao longo de seis semanas tendo como principais funções sedimentar os conteúdos estudados em aulas presenciais e proporcionar aos alunos a oportunidade de realizarem trabalhos de forma colaborativa utilizando ferramentas digitais. Assim, para além de um espaço de partilha de recursos didáticos, a plataforma serviu para a produção de artefactos colaborativos.

Assumindo-se como particularística, esta investigação enquadrou-se na tipologia de estudo de caso, debruçando-se deliberadamente sobre esta contingência específica (COUTINHO, 2013). Supondo-se ser única ou especial, o seu estudo pretendeu descobrir a que há nela de mais essencial e característico e, desse modo, contribuir para a compreensão global de um certo fenômeno de interesse (PONTE, 1994), neste caso concreto, o estudo e a aprendizagem colaborativa online em comunidades Do-It-Youself.

Os instrumentos de recolha de dados utilizados para tal fim foram os seguintes:

- Diário de bordo, onde foram relatadas as ocorrências mais relevantes a partir de notas sobre o desenvolvimento dos trabalhos em cada sessão.

- Questionário inicial e questionário final, a partir do qual foram recolhidas as opiniões dos alunos sobre a aprendizagem colaborativa e sobre a utilização de ferramentas digitais.

- Interações verbais, que consistiram na recolha, análise, redução e codificação das publicações e comentários dos alunos em discussões assíncronas.

- Quiz, a partir do qual se aferiu, por meio de um pequeno questionário, a compreensão dos alunos sobre as diferentes fases da metodologia projetual. 
- Tarefa, de caráter individual, consistiu na proposta de uma atividade de pesquisa sobre dobragens em papel.

- Entrevistas por grupo focal, nas quais os alunos refletiram sobre as suas aprendizagens.

- Vídeos tutoriais, ou artefactos feitos em grupo, que possibilitaram a avaliação do sucesso do trabalho colaborativo, tendo em conta a sua eficácia perante a comunidade.

A variedade dos instrumentos aplicados teve como intenção englobar as diferentes perspetivas dos participantes e, por outro lado, obter várias "medidas" dos fenômenos em análise, criando condições para triangular os dados e assegurar a credibilidade do estudo de caso (COUTINHO, 2015).

\section{Resultados e discussão}

Apesar de considerarmos a criação e a partilha dos vídeos o corolário do esforço colaborativo empreendido, há a referir que na sua concepção foram mobilizadas várias competências adquiridas ao longo do processo, quer no contacto e trabalho com ferramentas digitais, quer na consciencialização da comunidade como um meio de partilha de tutoriais enquanto traço distintivo das comunidades de aprendizagem Do-It-Yourself.

No diário de bordo, surgiram vários relatos da motivação demonstrada pelos intervenientes. Aos mecanismos interativos proporcionados pelo Edmodo foi-se acrescentando o acesso a ferramentas colaborativas, nomeadamente através dos documentos Google e de ferramentas de edição de vídeo do Youtube. Isso aconteceu na mesma medida que a comunidade se foi organizando no decorrer das semanas em grupos de trabalho. A troca de mensagens e comentários indicou que os alunos se envolveram numa primeira fase de forma cooperativa para, mais tarde, demonstrarem atividade colaborativa à medida que a formação de objetivos partilhados se tornou mais evidente. 
No questionário inicial e no questionário final as opiniões em relação à aprendizagem colaborativa são positivas, o mesmo acontece em relação às opiniões sobre a utilização de ferramentas digitais do Edmodo e sobre a aprendizagem em grupo.

As interações verbais foram recolhidas e medidas de forma estruturada, a aplicação do modelo de análise das discussões assíncronas online de Murphy (2004) confirmou algumas de nossas conclusões, nomeadamente que "os participantes se envolvem principalmente em processos relacionados com a presença social e o articular de perspetivas individuais" (2004, p. 430). Conseguimos atingir os níveis superiores de colaboração - "construir objetivos partilhados" e "produzir artefactos comuns" - embora com um número de entradas residual. Verificamos que, para atingir estes níveis, é imperativo que os objetivos e a indicação do produto final pretendido sejam bem explícitos. A análise dos resultados em cada semana sugere alguma correspondência entre os indicadores obtidos e o cronograma pré-estabelecido, o que não é de todo surpreendente tendo em conta o trabalho de scaffolding a empreender pelo moderador, que como Murphy (2004) indica é essencial para "levar os intervenientes numa discussão assíncrona online além da interação até à colaboração" (p. 422).

A resolução do quiz e da tarefa possibilitaram por um lado a aquisição de conhecimentos quanto à compreensão da metodologia projetual nas suas diferentes fases (MUNARI, 1981) e respectiva aplicação prática, e, por outro lado, o aprofundamento dos seus conhecimentos sobre origami. Ambas as atividades foram concretizadas online e tiveram índices de participação elevados, levando a que os alunos aprendessem não só com as suas realizações, mas também através dos contributos dos colegas.

As entrevistas por grupo focal permitiram perceber de que forma os alunos se adaptaram ao Edmodo e aos conteúdos e às ferramentas digitais disponibilizadas. Ao instigar os entrevistados a fazer uma autoavaliação do seu envolvimento nas tarefas, das possíveis dificuldades que estivessem a sentir e a falarem sobre as ferramentas que podiam usar para cumprir com aproveitamento o trabalho de grupo - tutorial de origami -, os alunos expressaram como tencionavam dividir as tarefas pelos 
elementos do grupo, entre outros aspetos importantes, como o material a usar para a captação da imagem e do áudio.

Quantos aos vídeos tutoriais, a leitura dos dados indica que o envolvimento no trabalho variou consoante os grupos, mas globalmente os resultados foram bastante positivos já que estes artefactos digitais foram apresentados e efetivamente usados como tutoriais cumprindo a sua principal função. Dessa dinâmica resultaram fotografias das dobragens em papel que cada um tentou fazer a partir dos vídeos. Os comentários que se geraram a partir dessas tentativas contribuíram para que a avaliação fosse feita pela comunidade como um todo e foram indicados pontos fortes e pontos a melhorar ou menos explícitos em cada tutorial. Os utensílios e ferramentas usados pelos alunos variaram de acordo com o quão à vontade se sentiam com as mesmas e os recursos que conseguiram mobilizar. Foram usadas câmaras digitais, mas sobretudo os telemóveis como meio de captação dos vídeos, e as ferramentas de edição utilizadas foram desde aplicativos dos próprios telemóveis, ao Windows Movie Maker e o Youtube Editor.

\section{Conclusões}

A investigação qualitativa envolve um processo de recolha de dados extremamente demorado, tendo em conta a grande quantidade de dados descritivos que há que reduzir e interpretar (YIN, 1994). Deparamo-nos com a necessidade de recolher e organizar as mensagens e comentários, transcrever as entrevistas a partir de gravações de áudio e organizar e refletir sobre uma grande variedade de dados, recolhidos a partir de instrumentos diversos.

No que concerne aos recursos materiais, o desenvolvimento desta investigação implicou a utilização de computadores com ligação à Internet, o que nem sempre foi possível assegurar em quantidade suficiente e com o software necessário, referimonos mais especificamente às aulas em que os alunos fizeram o questionário final, dado o número insuficiente de computadores na sala de aula em que foi realizado. Esta situação gerou um menor acompanhamento nas dúvidas que os alunos iam colocando, e alguma agitação. 
Considera-se que a técnica de análise de conteúdo, mesmo orientada por categorias bem definidas, poderá conter algum teor de subjetividade. A leitura de cada mensagem e atribuição de um significado e respectiva categorização poderá ser condicionada pela vivência de quem a analisa.

A credibilidade num estudo de caso não tem a ver com a representatividade das amostras, nem tampouco com a preocupação do investigador em controlar as várias fontes de invalidade associadas aos estudos quantitativos de tipo experimental (COUTINHO, 2013). Procuramos alcançá-la através de um trabalho continuado, envolvendo o estudo intenso e prolongado no seio do grupo, através do chamado prolonged engagement (GUBA \& LINCOLN, 1994), amiúde materializado através da descrição maciça, compacta, dos dados recolhidos, as thick descriptions (Stake, 1995).

A generalização dos resultados do estudo, ou a validade externa, que é um aspecto central nos estudos quantitativos, emerge aqui no contexto da transferibilidade, ou seja, a possibilidade dos resultados obtidos num determinado contexto, numa pesquisa qualitativa, poderem ser aplicados noutro contexto. (COUTINHO, 2015)

A necessidade de delimitar o corpo de dados, dada a quantidade de informação condicionou a possível profundidade no estudo, mas contribuiu, simultaneamente para a identificação de eventuais linhas de investigação a realizar futuramente.

A recolha e análise dos dados sustentam que uma comunidade DIY em contexto escolar pode potenciar aprendizagens colaborativas. Os alunos revelaram grande motivação em aprenderem em conjunto de forma cooperativa, ao partilharam e comentarem recursos descobertos na Internet, e também de forma colaborativa, ao realizarem os vídeos tutoriais sobre dobragens em papel.

A tenra idade dos alunos, que constituíram o público-alvo da investigação, exigiu uma presença muito forte do moderador que acompanhou de muito perto a evolução dos trabalhos. Apesar de considerarmos que idealmente a recolha e o tratamento dos dados deveriam ocorrer num horizonte temporal muito mais alargado, 
ficamos satisfeitos com a quantidade e a qualidade da informação recolhida, que foi demonstrativa da grande apetência, motivação e envolvimento dos alunos neste tipo de projetos.

Tendo em conta que o estudo foi realizado recorrendo a uma amostragem não probabilística: recorremos a uma turma já constituída (amostragem por conveniência), poderá ser difícil generalizar as conclusões para além do grupo em estudo (SCHUTT, 1999), contudo nada impede que os resultados sejam tidos na sua aplicação com outros discentes e em outras escolas. Considerando que a investigação foi levada a cabo em contexto real, há padrões de comportamento e trabalho que podem ser levados em conta para futuras investigações.

O facto de a maior parte das atividades ter sido realizada através da Internet e com um grau de autonomia considerável, implicou que nem sempre foi possível avançar como previsto no cronograma estabelecido, gerando pequenas flutuações no tempo. Apesar de pequenos contratempos, conseguimos levar a cabo a maior parte do trabalho no tempo estipulado, o que em muito se deveu a um planeamento rigoroso e a alguma adaptação perante situações não previstas.

\section{Referências}

ACKERMANN, E. Cultures of creativity and modes of appropriation: From DIY (Do It Yourself) to BIIT (Be In It Together). Retirado de: <http://cache.lego.com/r/legofoundation//media/LEGO\%20Foundation/Downloads/Cultures\%20of\%20Creativity/Edith\%20K\%20Ackermann.pdf >. 2013.

COUTINHO, C. P. Avaliação da qualidade da investigação qualitativa: algumas considerações teóricas e recomendações práticas. In Francislê Neri de Sousa et al. (Org.). Investigação Qualitativa: Inovação, Dilemas e Desafios, Volume 2, 1.a edição, p. 101-121. Oliveira de Azeméis: Ludomedia. 2015.

COUTINHO, C. P. Metodologia de Investigação em Ciências Sociais e Humanas. Coimbra: Almedina. 2013.

DILLENBOURG, P. What do you mean by collaborative learning? In: P. Dillenbourg (Ed.) Collaborativelearning: Cognitive and Computational Approaches, pp. 1-19. Oxford: Elsevier. 1999.

GUBA, E. G., \& LINCOLN, Y. S. Competing paradigms in qualitative research. In: N. K., Denzin, \& Y. S., Lincoln (Eds.), Handbook of qualitative research, pp. 105-117. Thousand Oaks, CA: Sage. 1994.

HARASIM, L. M. Learning Theory and online Technologies. New York: Routledge. 2012.

ITO, M., Baumer, S., Bittanti, M., Boyd, D., Cody, R., Herr, B. \& Tripp, L. Hanging out, messing around, geeking out: Living and learning with new media. Cambridge, England: MIT Press. 2009.

JENKINS H.; KNOBEL, C.; \& LANKSHEAR, M. (Eds.). DIY media. Creating, sharing and learning with new technologies, p. 231-253. New York: Peter Lang. 2010. 
KAFAI, Y. \& PEPPLER, K. Youth, Technology, and DIY: Developing Participatory Competencies. in Creative Media Production. In: WORTHAM, S. (Ed.), Youth Cultures, Language, and Literacy - Review of Research in Education, pp. 89-119. London: Sage. 2011.

KAMENETZ, A. DIY U: Edupunks, Edupreneurs, and the Coming Transformation of Higher Education. White River Junction. VT: Chelsea Green Publishing. 2010.

KUZNETSOV, S., \& PAULOS, E. Rise of the expert amateur: DIY projects, communities, and cultures. Retirado de: <http://portal.acm.org/citation.cfm?id=1868914.1868950\&amp;coll=DL\&amp;dl=GUIDE\& amp;CFID=10335082>. 2010.

LISBÔA, E. \& COUTINHO, C. P. Colaboração Online: Como Avaliar? Revista Paidéi@. Unimes Virtual, Volume 4. Disponível em: <http://revistapaideia.unimesvirtual.com.br>. 2013.

MUNARI, B. Das coisas nascem coisas. São Paulo: Martins Fontes. 1981.

MURPHY, E. Recognising and promoting collaboration in an online asynchronous discussion. British Journal of Educational Technology, Volume 35, Number 4, p. 421-431. Retirado de: <http://www.ucs.mun.ca/ emurphy/bjet_401.pdf>. 2004.

ROSCHELLE J. \& TEASLEY S. The construction of shared knowledge in collaborative problem solving. In: O'Malley, C (Ed.). Computer supported collaborative learning, p. 69-97. Berlin: Springer-Verlag. 1995.

STAKE, R. E. (1995). The Art of Case Study Research. Thousand Oaks, CA: Sage.

SCHUTT, R. Investigating the social world: the process and practice of research, Thousand Oaks, CA: Pine Forge Press. 1999.

VAN BOXTEL, C., VAN DER LINDEN, J.L., \& KANSELAAR, G. Collaborative learning tasks and the elaboration of conceptual knowledge, Learning and Instruction, Volume 10, Issue 4, p. 311-330. Disponível em: <https://www.kanselaar.net/wetenschap/files/colllearning_Carla_L\&l2000.pdf>. 2000.

YIN, R. Case Study Research: Design and Methods. Thousand Oaks, CA: Sage. 1994.

\section{Vitor Silva}

Mestre em Gestão de Sistemas de E-Learning pela Faculdade de Ciências Sociais e Humanas da Universidade Nova de Lisboa. Licenciado no Curso de Ensino da Educação Visual e Tecnológica pela Escola Superior de Educação do Instituto Politécnico de Viana do Castelo. Professor do Ensino Básico.

E-mail: vitor.fgf.silva@edu.azores.gov.pt

\section{Clara Coutinho}

Doutora em Ciências da Educação na especialidade de Tecnologia Educativa pela Universidade do Minho. Professora Auxiliar do Departamento de Estudos Curriculares e Tecnologia Educativa do Instituto de Educação da Universidade do Minho.

E-mail: ccoutinho@ie.uminho.pt

Currículo: https://sites.google.com/site/ccoutinhoum/ 